\title{
COMPARING THE ACCURACY OF GNSS POSITIONING VARIANTS FOR UAV BASED 3D MAP GENERATION
}

\author{
H. Haddadi Amlashi, F. Samadzadegan, F. Dadrass Javan*, M. Savadkouhi
}

Department of Surveying and Geomatics Engineering, University College of Engineering, University of Tehran, Tehran, Iran \{hesam.haddadi.am, samadz, fdadrasjavan,m.savadkouhi\}@ut.ac.ir

\author{
Commission I, WG I/2
}

KEY WORDS: GNSS, Post Process Kinematic, UAV, Multiple constellation.

\begin{abstract}
:
GNSS stands for Global Navigation Satellite System and is the standard generic term for satellite navigation systems that provide autonomous geo-spatial positioning with global coverage. The advantage of having access to multiple satellites is accuracy, redundancy, and availability at all the times. Though satellite systems do not often fail, if one fails GNSS receivers can pick up signals from other systems. If the line of sight is obstructed, having access to multiple satellites is also a benefit. GPS (Global Positioning System, USA), GLONASS (Global Navigation Satellite System, Russia), BeiDou (Compass, China), and some regional systems are positioning systems that are usually used. In recent years with the development of the UAVs and GNSS receivers, it is possible to manage an accurate PPK (Post Processing Kinematic) networks with a GNSS receiver mounted on a UAV to achieve the position of images principal points WGS1984 and to reduce the need for GCPs. But the most important challenge in a PPK task is, which a combination of different GNSS constellations would result in the most accurate computed position in checkpoints. For this purpose, this study focused on a PPK equipped UAV to map an open pit (Golgohar mine near Sirjan city). For the purpose, different combination of GPS, GLONASS and BeiDou used for position computed. Results are plotted and compared and found out having access to multiple constellations while doing a PPK task would bring higher accuracies in building photogrammetric models although it may cause some random error due to the higher values of noise while the number of the satellites increases.
\end{abstract}

\section{1- INTRODUCTION}

Drone systems that are used in photogrammetry are costeffective and provide outstanding flexibility in terms of where, when, and how to capture $2 \mathrm{D}$ and $3 \mathrm{D}$ data in comparison with airborne and satellite-based photogrammetry. Numerous researches in this area have been done by now (Haarbrink et al., 2006, Everaerts, J. et al., 2008, Sean P.Bemisa et al., 2011).

For years, just one GNSS system was available - the Global Positioning System - and just one fully available signal on the L1 frequency. Eventually, some studies discovered how to exploit certain characteristics of the encrypted L2 signal to come up with so-called codeless and semi-codeless techniques that enabled dual-frequency positioning (Grewal, M. S. et al., 2007). This hastened the development of user equipment that used the carrier phase of signals as well as the code to deliver high-precision results. Today, the airwaves are full of many kinds of GNSS signals on many different frequencies. The simplest kinds of receivers such as those found in mobile phones, however, still use just one frequency, L1. But as applications move up the value chain of accuracy, even more, sophisticated signal processing techniques are appearing in more types of GNSS receivers both single- and dual-frequency. The GNSS receivers are divided into two categories as single frequency and dualfrequency systems and also they can track one or multiple constellations. Dual-frequency and multiple constellation mode are more accurate than other GNSS receivers. In a case of a rare event if one of the GNSS systems fails the GNSS receiver can track other constellation for accurate 
positioning. That's why we used a dual-frequency multiple constellation mode GNSS receiver in this study.

The initial GNSS receivers were too big to be mounted on UAVs, so we had to have GCPs and use triangulation for improving a model accuracy in photogrammetric projects. But in recent years, by developing new GNSS receiver technology, that can be easily mounted on a UAV, we can achieve direct geo-referencing elements with high accuracy in photogrammetric image blocks with no GCPs.

Mountable GNSS receivers now can make UAVs more applicable like automatic take-off, landing, auto modes for capturing images, and acquiring UAV attitude during the flight and can provide us with high accuracy in positioning information which can result in more precise bundle adjustment.

GNSS correction technology enhances the quality of location data by using global navigation satellite system receivers to generate precise and accurate data. It's a technology that's been used with different tools on the ground for years. Only recently it has been offered as an addon to a survey drone for better survey-grade photogrammetry. There are currently two technologies driving the conversation around GNSS receiver's correction technology - Real-Time Kinematic (RTK) and Post Processing Kinematic (PPK):

- Real Time Kinematic (RTK) is a GNSS correction technology that provides real-time correction solution to position data.

- $\quad$ Post Processed Kinematic (PPK), GNSS correction technology that works to correct position data after the data has been logged.

The result of using any of these systems is a geo-referenced aerial image block, which can be directly used for further photogrammetric process as point cloud, digital 3D earth models, and orthophoto mosaics generation.

M. Abd Rabbou et al., 2016 assessed the accuracy analysis GNSS based photogrammetric blocks (M. Abd Rabbou et al., 2016) for PPP (Precise Point Positioning) applications. They developed a new GNSS PPP model, which combines the observations of GPS, GLONASS, Galileo, and BeiDou satellite navigation systems for precise applications. In their study, both un-differenced and between-satellite singledifference (BSSD) ionosphere-free GNSS PPP models are developed. The results indicate that both GPS and GPS/Galileo PPP present comparable positioning accuracy levels, which is attributed to the limited number of Galileo satellites.

Different constellations in static modes and observations for positioning have been analyzed which is believed theoretically, there are some errors and biases that can be eliminated or subtracted by the strength of satellite geometric (Khomsinl et al., 2019). One factor to get a good satellite geometry is to increase the number of satellites received by the receiver. The development of receiver technology is currently able to capture GPS, GLONASS, and BeiDou signals at one time. Thus the receiver can receive many satellites and finally the shape of the geometric satellite becomes better. The (GPS + GLONASS + BeiDou) and (GPS + GLONASS) strategy have the same horizontal and vertical accuracies and All strategies have horizontal accuracies less than or equal to $1 \mathrm{~cm}$ and vertical accuracies less than $2 \mathrm{~cm}$ for baselines $\leq 5 \mathrm{~km}$.

In the case of direct geo-referencing, some methods used a different combination of GPS/IMU components (Stöcker et al., 2017). The development of high-quality Inertial Measurement Units (IMU) and GNSS technology and dedicated RTK and PPK solutions for UAVs promise accurate measurements of the exterior orientation (EO) parameters that allow geo-reference of the captured images. The study assesses the final geometric accuracy using direct geo-referencing with high-quality post-processed IMU/GNSS and PPK corrections and the results show that the use of the post-processed GNSS and IMU data was particularly beneficial to enhance the image orientation quality.

Hongxing Sun et al., 2016 reviewed multi-mode GNSS positioning and it's applications in large scale photogrammetry (Hongxing Sun et al., 2016). The precise multimode GNSS positioning for UAV and its application in large scale photogrammetry is studied. They use multimode GNSS instead of single GPS constellation to resolve the three exterior line elements of each image. With the multiconstellation system, the number of visible satellites is significantly increased and the geometry distribution of the satellites is well improved. The positioning accuracy and robustness are therefore getting better compared to GPS positioning.

This study aims to discuss whether different combinations of GNSS constellations impact $3 \mathrm{~d}$ accuracy of bundle adjustment based on checkpoints and which combination will result in higher accuracy.

\section{2- MATERIALS AND METHOD}

The ability of a GNSS receiver to handle multiple frequencies from multiple constellations in the calculation of position is essential to gain optimal error resolution. Using multi-frequency receivers is the most effective way to remove Ionospheric error from the position calculation (equation 1). The ionospheric error varies with frequency so it impacts the various GNSS signals differently. 
$\Delta L_{A B}^{j} \equiv L_{A}^{j}-L_{B}^{j}$

$=\left(\rho_{A}^{j}+C T_{A}-C T^{J}+Z_{A}^{j}-I_{A}^{j}+B_{A}^{j}\right)-\left(\rho_{B}^{j}+C T_{B}-C T^{J}+Z_{B}^{j}-I_{B}^{j}\right.$

$$
\left.+B_{B}^{j}\right)
$$

$=\left(\rho_{A}^{j}-\rho_{B}^{j}\right)+\left(C T_{A}-C T_{B}\right)-\left(C T^{J}-C T^{J}\right)+\left(Z_{A}^{j}-Z_{B}^{j}\right)-\left(I_{A}^{j}-I_{B}^{j}\right)-\left(B_{A}^{j}\right.$ $\left.-B_{B}^{j}\right)$

$=\Delta \rho_{A B}^{j}+c \Delta T_{A B}+\Delta Z_{A B}^{j}-\Delta I_{A B}^{j}+\Delta B_{A B}^{j}$

By comparing the delays of two GNSS signals, L1 and L2, for example, the receiver can correct the impact of Ionospheric errors. Multi-frequency receivers also provide more immunity to interference. If there is interference in the L2 frequency band around $1227 \mathrm{MHz}$, a multi-frequency receiver will still track L1 and L5 signals to ensure ongoing positioning. A multi-constellation receiver can access signals from several constellations Like GPS, GLONASS, BeiDou. The use of other constellations in addition to GPS results in being a larger number of satellites in the field of view, which results in the following benefits:

- Reduction in signal acquisition time.

- Improved position and time accuracy.

- Reduction of problems caused by obstructions such as buildings and foliage.

- Improved spatial distribution of visible satellites, resulting in improved dilution of precision and satellites geometry.

To determine a position in GNSS-only mode, a receiver must track a minimum of four satellites. In multi-constellation mode, the receiver must track five satellites, at least one of which must be from a satellite in the other constellation, so the receiver can determine the time offset between constellations. So in this way, we can assure that we will have a solid solution in our positioning and it will be fixed. Alcantarilla et al., 2006 reviewed the benefits of multiconstellation GNSS augmentations. It showed that the multiconstellation system concept proposed integrating the global navigation satellite systems (GPS, GLONASS, GALILEO) in one common interface to make the user profit from enhanced capabilities (Alcantarilla et al., 2006).

In this study, a fixed-wing UAV is selected to acquired images over Golgohar mine. Achieved images and positioning data are then processed to extract the position of principal points of images with RTKLib in different scenarios as follows:
I. Beidou
II. GLONASS
III. GPS
IV. GLONASS, Beidou
V. $\quad$ GPS, Beidou

\author{
VI. GPS, GLONASS \\ VII. GPS, GLONASS, Beidou
}

Then final precision is assessed based on checkpoints which they are evenly dispersed in the area of study and are compared over the parameters of:

- Highest number of satellites

- Quality of the solution

- Minimum time to fix solution

- Precision including:

$\Delta$ Latitude

$\Delta$ Longitude

$\Delta$ Height

- $\quad$ Signal to noise ratio

Data collection encompassed UAV data acquisition and PPK-GNSS of 15 evenly spaced checkpoints in the area. 800 images were taken and the altitude was set to 300 meters with GSD of $3 \mathrm{~cm}$ and also one cross flight was conducted to improve the stability of bundle adjustment of the photogrammetry block. The camera shot was set for every 32 meters which resulted in an overlap of $\% 80$ and sidelap of $\% 70$. The GNSS receiver was a dual-frequency PPK system with a $20 \mathrm{~Hz}$ rate output of raw data with frequencies GPS L1/L2, GLONASS L1/L2, BeiDou B1/B2. Then the measurements of the points converted to Rinex 3.02 with RTKLib and the images were geotagged with the camera and GPS $\log$ in each scenario.

The study took place in Golgohar mine near Sirjan city. The lowest height is $1529 \mathrm{~m}$ and the highest is $1805.13 \mathrm{~m}$. Figure 1 presents an overview of the test area.

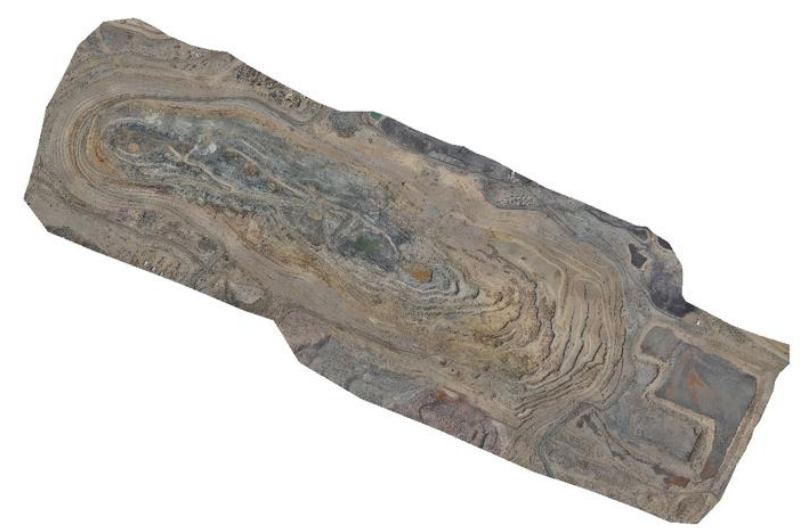

Figure 1. overview of the test area

The whole block was imaged with Sony alpha 6000 camera that the specification is presented in table 1 .

\begin{tabular}{ll}
\hline Feature & Description \\
\hline Lens Mount & Sony E \\
\hline Focal length & $35 \mathrm{~mm}$ \\
\hline Focal Length (actual) & $16 \mathrm{~mm}$ \\
\hline Zoom Ratio & $3.31 \mathrm{x}$ \\
\hline
\end{tabular}




\begin{tabular}{ll}
\hline Aperture Range & $\begin{array}{l}\text { f/3.5 7-blade circular } \\
\text { aperture }\end{array}$ \\
\hline
\end{tabular}

Table 1. Camera specification

GNSS receiver Tersus is used as PPK receiver. The system specifications and visualizations are presented in Table 2.

\begin{tabular}{ll}
\hline Feature & Description \\
\hline $\begin{array}{l}\text { Signal tracking for } \\
\text { primary antenna }\end{array}$ & GPS L1/L2, GLONASS \\
\hline $\begin{array}{l}\text { Signal tracking for } \\
\text { secondary antenna }\end{array}$ & GPS L1+GLONASS L2 or \\
\hline GNSS channels & GPS L1+BeiDou B2 \\
\hline Single point positioning & Horizontal $1.5 \mathrm{~m}$ \\
accuracy (RMS) & Vertical $3 \mathrm{~m}$ \\
\hline PPK positioning accuracy & Horizontal10 mm $+1 \mathrm{ppm}$ \\
(RMS) & Vertical $15 \mathrm{~mm}+1 \mathrm{ppm}$ \\
\hline $\begin{array}{l}\text { Observation accuracy } \\
\text { (Zenith direction) }\end{array}$ & C/A Code $10 \mathrm{~cm}$ \\
\hline Time to first fix (TTFF) & P Code $10 \mathrm{~cm}$ \\
\hline Timing accuracy (RMS) & Corrier phase $1 \mathrm{~mm}$ \\
\hline Velocity accuracy (RMS) & Warm start $<30 \mathrm{~s}$ \\
\hline Initialization (Typical) & $0.03 \mathrm{~m} / \mathrm{s}$ \\
\hline Initialization reliability & $<10 \mathrm{~s}$ \\
\hline \multicolumn{2}{c}{ Table 2. GNSS receiver specification } \\
\hline
\end{tabular}

\section{3- DISCUSSIONS}

One of the important factors in positioning with GNSS receivers is the number of visible satellites in the time of the survey. The more the number of satellites is, the better the geometry would be and it will cause more accurate positioning results. It's obvious that if we use multiconstellation mode we have more satellites to track so the results of the positioning would become more accurate. In table 3 the number of satellites available in each scenario at the time of the survey is presented.

\begin{tabular}{cc}
\hline Scenarios & Satellites \\
\hline GPS & 7 \\
\hline GLONASS & 6 \\
\hline Beidou & 5 \\
\hline GLONASS + Beidou & 11 \\
\hline GPS + Beidou & 12 \\
\hline GLONASS + GPS & 13 \\
\hline GLONASS + GPS + Beidou & 18 \\
\hline
\end{tabular}

Table 3. Number of the available satellites

Another way of perceiving the number of satellites is the sky plot of the satellites at the time of the survey which can show how satellites move and which signals are logged at the time which can be seen in figure 2 . The green lines show satellite directions in which $\log \mathrm{L} 1$ and L2 signals and pink lines show only L2 signals which they are the identifier of the quality of the solution.

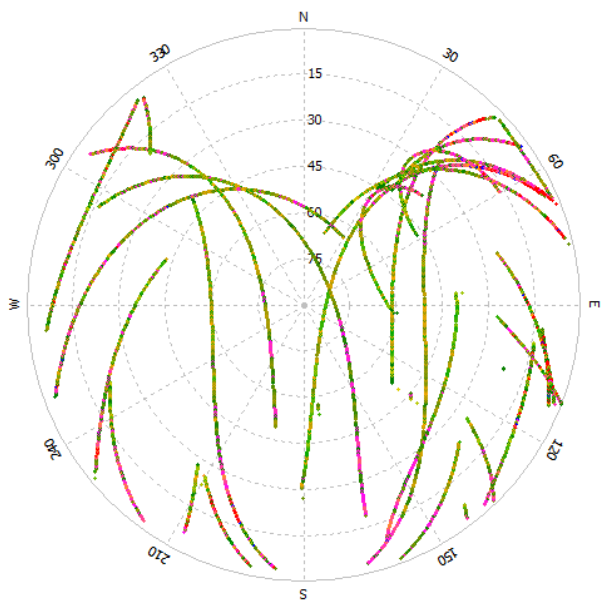

Figure 2. sky plot of GNSS receivers

Another important factor is the quality of the solution and how long does it take for constellation to be fixed. In table 4 the quality achieved and time needed for each of the Scenarios to get fixed is presented (Q1 represents fix and Q2 float).

\begin{tabular}{|c|c|c|}
\hline Scenarios & $\begin{array}{l}\text { Quality of the } \\
\text { solution }\end{array}$ & $\begin{array}{l}\text { Minimum } \\
\text { time to fix (s) }\end{array}$ \\
\hline $\begin{array}{l}\text { GPS + GLONASS } \\
+ \text { Beidou }\end{array}$ & $\begin{array}{l}\text { Q1: \% } 94 \\
\text { Q2:6 }\end{array}$ & 17.050 \\
\hline GPS + GLONASS & $\begin{array}{l}\text { Q1: } \% 83.7 \\
\text { Q2: } 16.3\end{array}$ & 29.550 \\
\hline GPS + Beidou & $\begin{array}{l}\text { Q1: \% 86.1 } \\
\text { Q2: } 13.9\end{array}$ & 27.250 \\
\hline $\begin{array}{l}\text { GLONASS + } \\
\text { Beidou }\end{array}$ & $\begin{array}{l}\text { Q1: \% 67.4 } \\
\text { Q2: } 32.6\end{array}$ & 33.150 \\
\hline GPS & $\begin{array}{l}\text { Q1: \% } 92.9 \\
\text { Q2: } 7.1\end{array}$ & 21.400 \\
\hline GLONASS & $\begin{array}{l}\text { Q1: \% 64.3 } \\
\text { Q2:\% } 26.7\end{array}$ & 35.210 \\
\hline Beidou & $\begin{array}{l}\text { Q1: \% } 70.9 \\
\text { Q2:\% } 29.1 \\
\end{array}$ & 32.350 \\
\hline
\end{tabular}

Table 4. Quality of the solutions, minimum time needed to get fixed

For the next factor, we studied the accuracy achieved for the evenly distributed checkpoints in the test area of study. Figure 5 presents the distribution of checkpoints. The error in latitude, longitude, and altitude values of the points are measured since we had the accurate location of the points from ground surveying.

The images were aligned using Agi soft metashape (https://www.agisoft.com) then dense point cloud was built and orthophoto and DEM were then extracted. In Figure 4 you can see the photogrammetric block of the mine. 


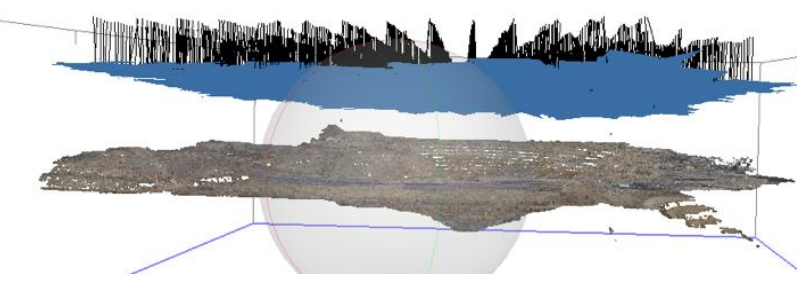

Figure 3. Photogrammetric block of Golgohar mine

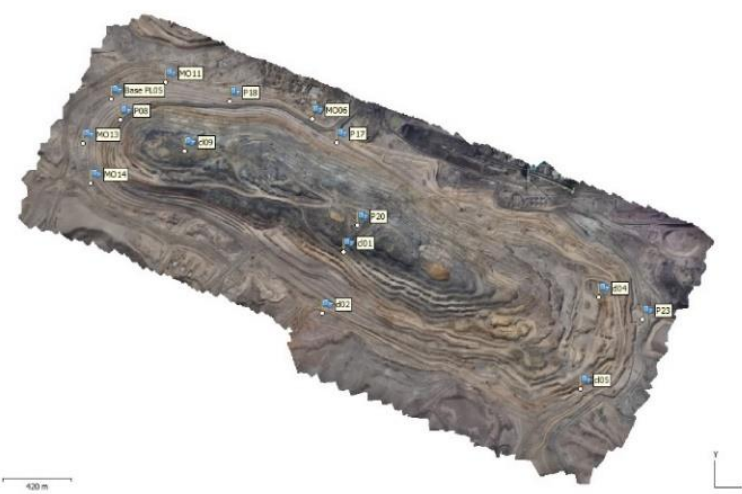

Figure 4. Distribution of check points in Golgohar mine on orthophoto

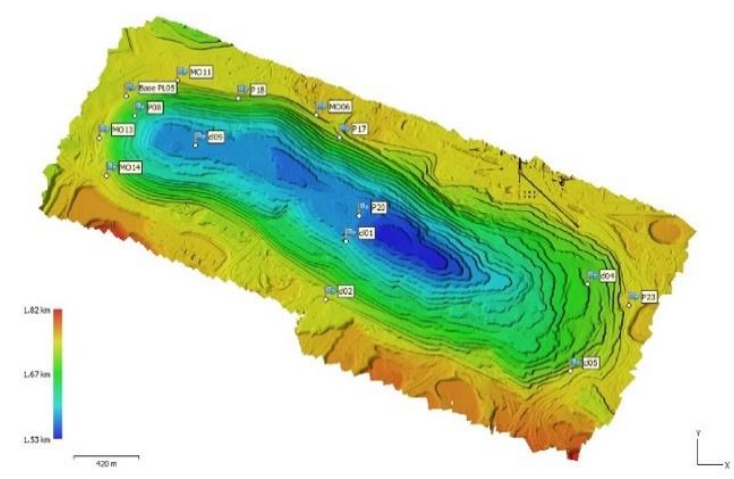

Figure 5. DEM of the Golgohar mine

The error measurements are in decimeter and the graphs below show the results in every checkpoint and every scenario is shown in different colors. (GPS: pale blue, GPS + Beidou: dark blue, GLONASS: orange, GLONASS + Beidou: green, Beidou: gray, GPS + GLONASS: yellow and GPS + GLONASS + Beidou: red). Checkpoints near the edge of the area have higher errors because of the photogrammetric bundle adjustment.

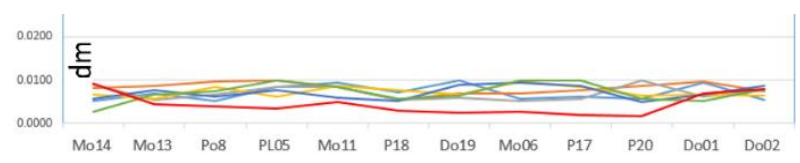

Figure 6. Latitude error

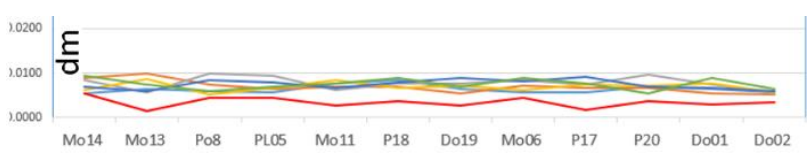

Figure 7. Longitude error

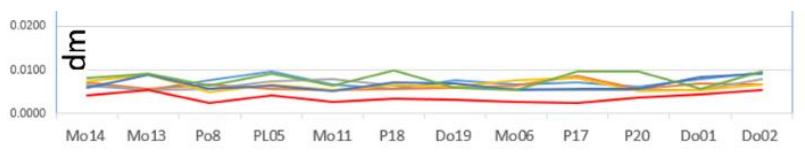

Figure 8. Height error

As it is obvious in the results, the only constellation that has higher accuracy is the first scenario where GPS, GLONASS, and Beidou all together are applied. So far the position accuracy had the best result when all constellation systems together are used and the last but not the least factor is signal to noise ratio. SNR is usually expressed in terms of decibels. It refers to the ratio of the signal power and noise power in a given bandwidth. The sources of white noise in a GNSS receiver are usually described by the antenna noise temperature and the receiver noise temperature. In figures 9 , 10,11 , and 12 SNR factor in different scenarios is studied. SNR in the 25 to 45 range is the best and it shows better signal power.

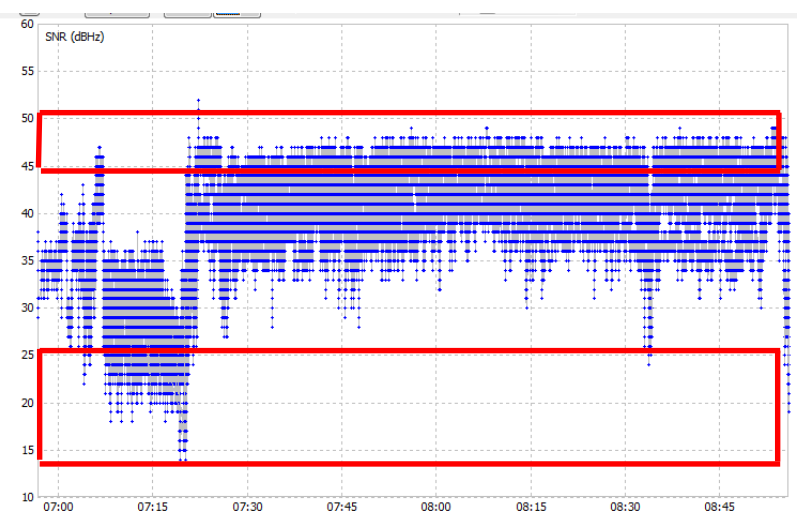

Figure 9. GPS signal to noise ratio

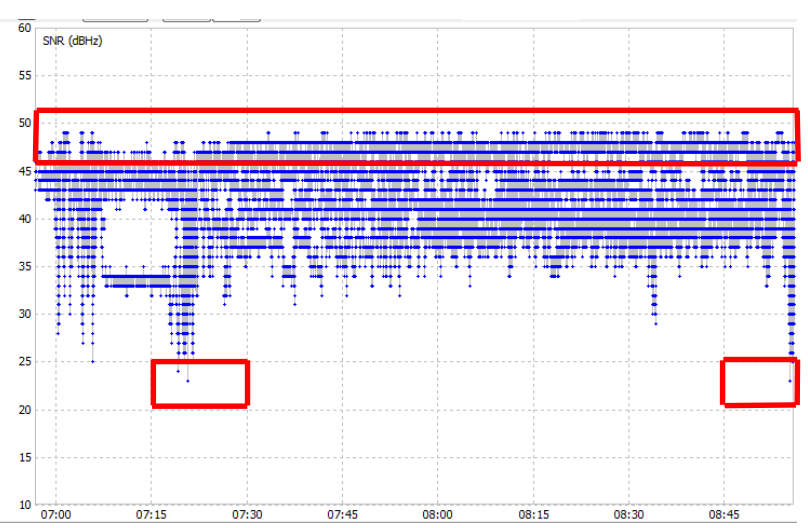

Figure 10. GlONASS signal to noise ratio 


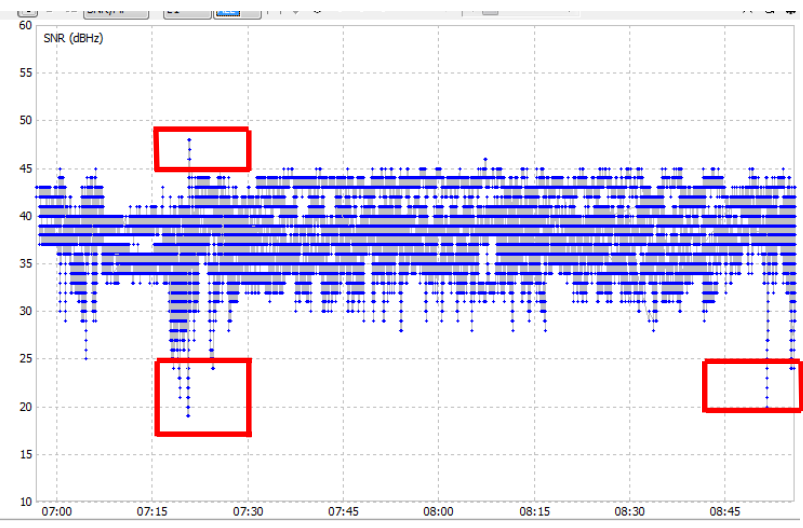

Figure 11. Beidou signal to noise ratio

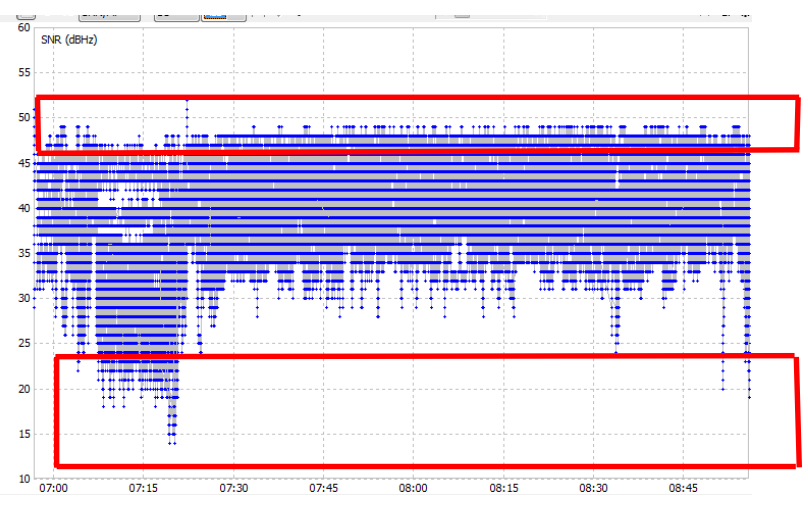

Figure 12. Hybrid signal to noise ratio

As it is obvious from the figures, the main problem is the in signal to noise ratio. When it is used in multi-constellation mode, the ratio will increase and it makes random error increase as long as the time of observation continues. The notable point here is that when only BeiDou is tracking, the SNR factor is very low due to its lower number of observable satellites. The highlighted red area shows obvious errors in SNR while tracking satellites.

\section{4- CONCLUSION}

In this study different factors in the accuracy of multiconstellation PPK tasks are reviewed. For the purpose, 7 scenarios as GPS, GLONASS, Beidou, dual combination, and GPS + GLONASS + Beidou are proposed. A photogrammetric block of images acquired by a fixed-wing UAV over an open-pit mine is used as the data set. The openpit mine is selected which enjoys over 300 meters' difference in height values. Achieved results can be summarized as:
- Accuracy of positioning in a PPK task in multi constellation mode is higher than single and dual mode constellation

- Quality of the solution in multi constellation mode is at its best value due to its best geometric diagram of satellites.

- Less time is needed for multi constellation GNSS receiver to get fixed and start logging than single and dual constellation mode.

- In more than one constellation mode random error may increase due to lower SNR value.

For further studies, the different accuracy in checkpoints between RTK and PPK in the multi-constellation mode in a survey Can be investigated to find out the difference between the scenarios.

\section{5- REFERENCES}

Abd Rabbou, M., \& El-Rabbany, A. 2017. Performance analysis of precise point positioning using multiconstellation GNSS: GPS, GLONASS, Galileo and BeiDou. Survey Review, 49-352, 39-50.

Alcantarilla, I., Porras, D., Tajdine, A., Zarraoa, N., \& Lévy, J. C. 2006. The benefits of multi-constellation GNSS augmentations. In Proceedings of the 19th International Technical Meeting of the Satellite Division of The Institute of Navigation - ION GNSS 2006, Fort Worth, TX pp. 930938.

Anjasmara, I. M., Pratomo, D. G., \& Ristanto, W. 2019. Accuracy Analysis of GNSS GPS, GLONASS and BEIDOU Obsevation For Positioning. In E3S Web of Conferences Vol. 94, p. 01019. EDP Sciences.

Bemis, S. P., Micklethwaite, S., Turner, D., James, M. R., Akciz, S., Thiele, S. T., \& Bangash, H. A. 2014. Groundbased and UAV-based photogrammetry: A multi-scale, high-resolution mapping tool for structural geology and paleoseismology. Journal of Structural Geology, 69, 163178.

Bonet, B., Alcantarilla, I., Flament, D., Rodriguez, C., \& Zarraoa, N. 2009. The benefits of multi-constellation GNSS: reaching up even to single constellation GNSS users. In Proceedings of the 22nd International Technical Meeting of The Satellite Division of the Institute of Navigation ION GNSS, Savannah, GA, USA pp. 22-25.

Cho, A., Kim, J., Lee, S., Choi, S., Lee, B., Kim, B., ... \& Kee, C. 2007. Fully automatic taxiing, takeoff and landing of a UAV using a single-antenna GPS receiver only. In 2007 
International Conference on Control, Automation and Systems pp. 821-825. IEEE.

Everaerts, J. 2008. The use of unmanned aerial vehicles UAVs for remote sensing and mapping. The International Archives of the Photogrammetry, Remote Sensing and Spatial Information Sciences, 37 2008, 1187-1192.

Kornfeld, R. P., Hansman, R. J., \& Deyst, J. J. 1998. Singleantenna GPS-based aircraft attitude determination. Navigation, 451, 51-60.

Stöcker, C., Nex, F., Koeva, M., \& Gerke, M. 2017. Quality assessment of combined IMU/GNSS data for direct georeferencing in the context of UAV-based mapping. The International Archives of Photogrammetry, Remote Sensing and Spatial Information Sciences, 42, 355.

Sun, H., Li, L., Ding, X., \& Guo, B. 2016. The precise multimode GNSS positioning for UAV and its application in large scale photogrammetry. Geo-spatial Information Science, 193, 188-194.

Ulusoy, İ., Şen, E., Tuncer, A., Sönmez, H., \& Bayhan, H. 2017. 3D Multi-view Stereo Modelling of an Open Mine Pit Using a Lightweight UAV. Türkiye Jeoloji Bülteni, 602, 223-242.

Wang, J., Garratt, M., Lambert, A., Wang, J. J., Han, S., \& Sinclair, D. 2008. Integration of GPS/INS/vision sensors to navigate unmanned aerial vehicles. The International Archives of the Photogrammetry, Remote Sensing and Spatial Information Sciences, 37part B1, 963-969. 